\title{
Contents
}

Entropy of Eigenfunctions

Nalini Anantharaman, Herbert Koch and Stéphane Nonnenmacher

$1 \quad$ Motivations . . . . . . . . . . . . . . . . . . . . . . . . . . 1

$2 \quad$ Main Result .................................. 4

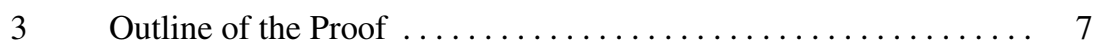

3.1 Definition of the Metric Entropy ............... 7

3.2 From Classical to Quantum Dynamical Entropy ........ 9

3.3 Entropic Uncertainty Principle................ 13

3.4 Applying the Entropic Uncertainty Principle to the

Laplacian Eigenstates ......................... 14

References ................................ 21

Stability of Doubly Warped Product Spacetimes $\ldots \ldots \ldots \ldots \ldots \ldots .23$

Lars Andersson

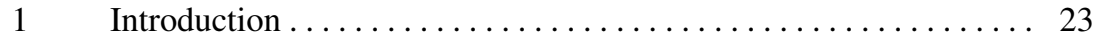

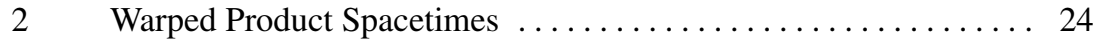

$2.1 \quad$ Asymptotic Behavior ...................... 26

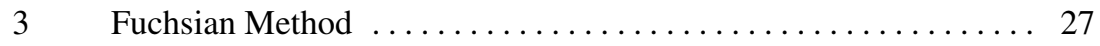

$3.1 \quad$ Velocity Dominated Equations................. 28

$3.2 \quad$ Velocity Dominated Solution .................. 29

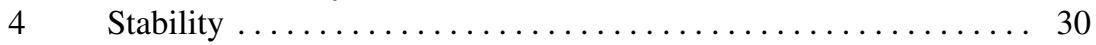

References ................................ 31

Rigorous Construction of Luttinger Liquids Through Ward Identities . . 33

Giuseppe Benfatto

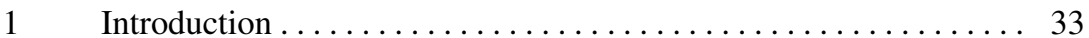

2 The Tomonaga Model with Infrared Cutoff . . . . . . . . . . . 34

3 The RG Analysis .............................. 35

$4 \quad$ The Dyson Equation ............................. 37

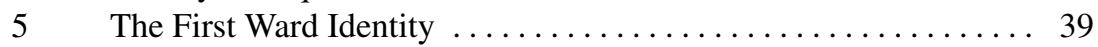

$6 \quad$ The Second Ward Identity $\ldots \ldots \ldots \ldots \ldots \ldots \ldots \ldots \ldots \ldots \ldots$ 
$7 \quad$ The Euclidean Thirring Model $\ldots \ldots \ldots \ldots \ldots \ldots \ldots \ldots \ldots \ldots 4$

References ................................. 43

New Algebraic Aspects of Perturbative and Non-perturbative Quantum

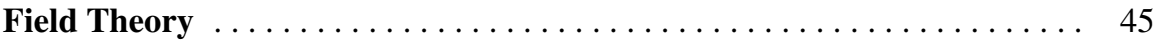

Christoph Bergbauer and Dirk Kreimer

$1 \quad$ Introduction .................................. 45

2 Lie and Hopf Algebras of Feynman Graphs $\ldots \ldots \ldots \ldots \ldots . \ldots 46$

3 From Hochschild Cohomology to Physics ............... 50

4 Dyson-Schwinger Equations ..................... 51

$5 \quad$ Feynman Integrals and Periods of Mixed (Tate) Hodge

Structures ................................... 55

References ............................... 57

Exact Solution of the Six-Vertex Model with Domain Wall Boundary

Conditions . . . . . . . . . . . . . . . . . . . . . . . . . . . . . . . 59

Pavel M. Bleher

$1 \quad$ Six-Vertex Model ............................. 59

2 Phase Diagram of the Six-Vertex Model ............... 62

3 Izergin-Korepin Determinantal Formula .............. 63

4 The Six-Vertex Model with DWBC and a Random Matrix

Model................................... 63

5 Asymptotic Formula for the Recurrence Coefficients ......... 65

$6 \quad$ Previous Exact Results ......................... 67

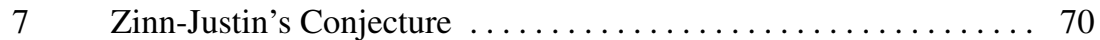

$8 \quad$ Large $N$ Asymptotics of $Z_{N}$ in the Ferroelectric Phase ....... 71

References ................................. 71

Mathematical Issues in Loop Quantum Cosmology . . . . . . . . . . 73

Martin Bojowald

$1 \quad$ Introduction .................................. 73

2 Quantum Representation and Dynamical Equations .......... 75

$2.1 \quad$ Quantum Reduction ......................... 75

$2.2 \quad$ Dynamics .............................. 76

3 Quantum Singularity Problem $\ldots \ldots \ldots \ldots \ldots \ldots \ldots \ldots \ldots \ldots \ldots$

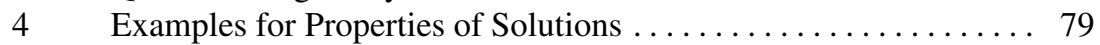

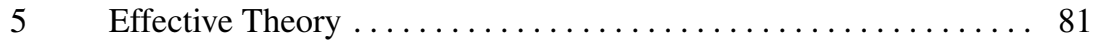

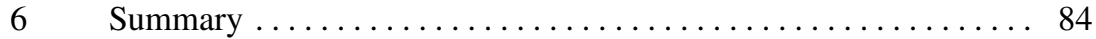

References .................................. 84

Boundary Effects on the Interface Dynamics for the Stochastic

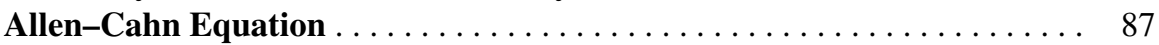

Lorenzo Bertini, Stella Brassesco and Paolo Buttà

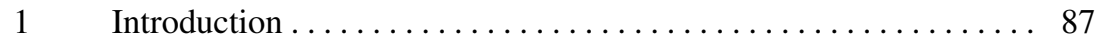

2 Results and Strategy of Proofs $\ldots \ldots \ldots \ldots \ldots \ldots \ldots \ldots \ldots$

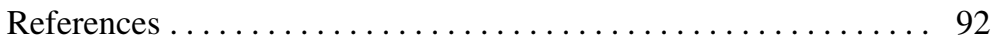


Dimensional Entropies and Semi-Uniform Hyperbolicity . . . . . . . . 95

Jérôme Buzzi

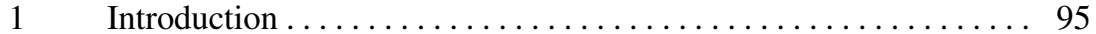

$2 \quad$ Low Dimension. ............................ 97

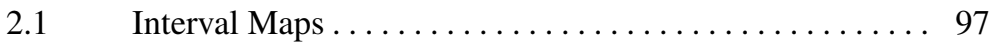

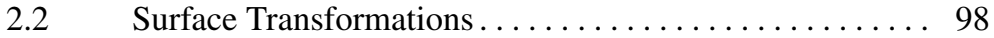

3 Dimensional Entropies . . . . . . . . . . . . . . . . . . . . . . . . . . . . . 99

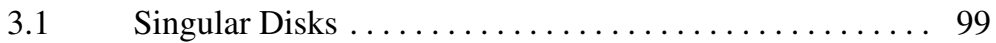

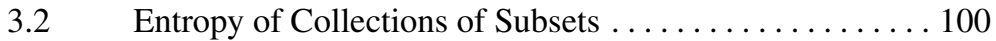

3.3 Definitions of the Dimensional Entropies . . . . . . . . . 101

4 Other Growth Rates of Submanifolds . . . . . . . . . . . . . . . . . . . . . . 102

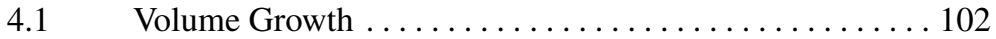

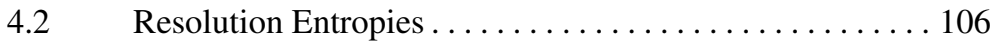

$5 \quad$ Properties of Dimensional Entropies . . . . . . . . . . . . . . . 107

$5.1 \quad$ Link between Topological and Resolution Entropies . . . 107

5.2 Gap Between Uniform and Ordinary Dimensional

Entropies ......................... 108

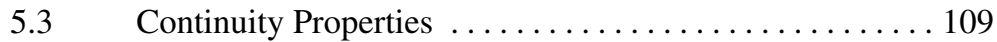

$6 \quad$ Hyperbolicity from Entropies . . . . . . . . . . . . . . . 110

$6.1 \quad$ A Ruelle-Newhouse Type Inequality . . . . . . . . . 110

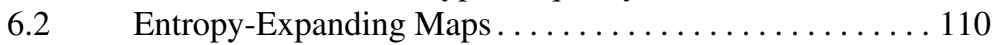

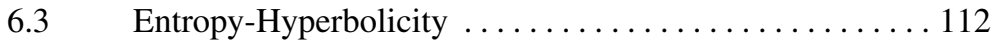

$6.4 \quad$ Examples of Entropy-Hyperbolic Diffeomorphisms . . . . 113

$7 \quad$ Further Directions and Questions . . . . . . . . . . . . . . 113

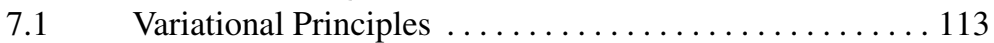

7.2 Dimensional Entropies of Examples ............ 113

$7.3 \quad$ Other Types of Dimensional Complexity . . . . . . . . . . 114

$7.4 \quad$ Necessity of Topological Assumptions . . . . . . . . . 114

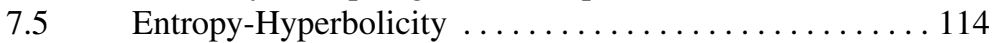

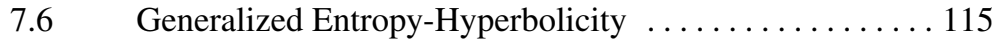

$8 \quad C^{r}$ Sizes . . . . . . . . . . . . . . . . . . . . . . . . . . . . 115

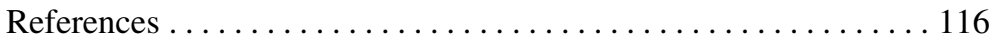

The Scaling Limit of (Near-)Critical 2D Percolation . . . . . . . . . . . . . . 117

Federico Camia

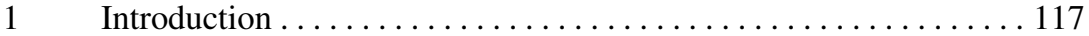

$1.1 \quad$ Critical Scaling Limits and SLE . . . . . . . . . . . 117

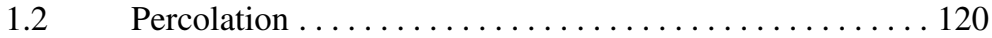

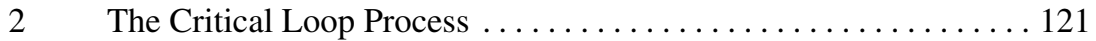

$2.1 \quad$ General Features . . . . . . . . . . . . . . . . . . . 121

$2.2 \quad$ Construction of a Single Loop . . . . . . . . . . . . . 122

3 The Near-Critical Scaling Limit . . . . . . . . . . . . . . . . 124

References ................................ 125 
Black Hole Entropy Function and Duality . . . . . . . . . . . . . . . . . 127

Gabriel Lopes Cardoso

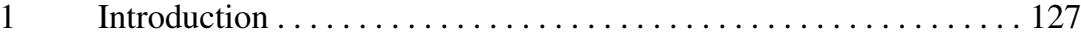

2 Entropy Function and Electric/Magnetic Duality Covariance . . . . 128

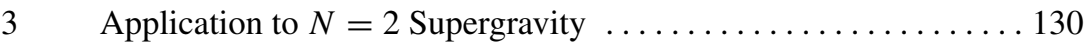

$4 \quad$ Duality Invariant OSV Integral $\ldots \ldots \ldots \ldots \ldots \ldots \ldots \ldots \ldots \ldots \ldots \ldots$

References ............................... 133

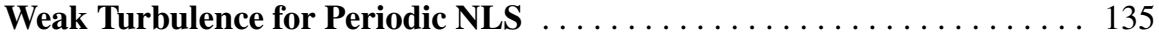

James Colliander

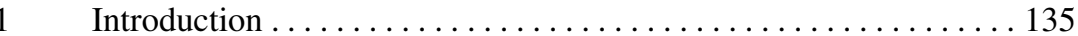

$2 \quad$ NLS as an Infinite System of ODEs . . . . . . . . . . . . 137

3 Conditions on a Finite Set $\Lambda \subset \mathbb{Z}^{2} \ldots \ldots \ldots \ldots \ldots \ldots \ldots$

4 Arnold Diffusion for the Toy Model ODE ............... . 139

$5 \quad$ Construction of the Resonant Set $\Lambda \ldots \ldots \ldots \ldots \ldots \ldots \ldots \ldots$

References ............................... 142

Angular Momentum-Mass Inequality for Axisymmetric Black Holes . . . 143 Sergio Dain

$1 \quad$ Introduction . . . . . . . . . . . . . . . . . . . . . . . . . . . 143

2 Variational Principle for the Mass . . . . . . . . . . . . . . . 144

References ............................... 147

Almost Everything About the Fibonacci Operator . . . . . . . . . . . . . . . . . 149

David Damanik

$1 \quad$ Introduction . . . . . . . . . . . . . . . . . . . . . . . . . . . . . . . 149

2 The Trace Map ................................. 150

3 The Cantor Structure and the Dimension of the Spectrum . . . . . . 152

4 The Spectral Type ............................ 154

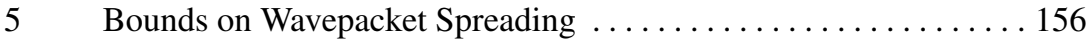

References ............................... 158

Entanglement-Assisted Quantum Error-Correcting Codes . . . . . . . . . . 161 Igor Devetak, Todd A. Brun and Min-Hsiu Hsieh

$1 \quad$ Introduction . . . . . . . . . . . . . . . . . . . . . . . 161

$2 \quad$ Notations . . . . . . . . . . . . . . . . . . . . . . 162

3 Entanglement-Assisted Quantum Error-Correcting Codes . . . . . . 163

3.1 The Channel Model: Discretization of Errors . . . . . . . . 164

3.2 The Entanglement-Assisted Canonical Code ......... 165

3.3 The General Case . . . . . . . . . . . . . . . . . . 167

$3.4 \quad$ Distance . . . . . . . . . . . . . . . . . . . . . . . . . . . . 169

3.5 Generalized $\mathbb{F}_{4}$ Construction . . . . . . . . . . . . . . . . 169

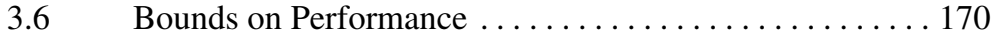

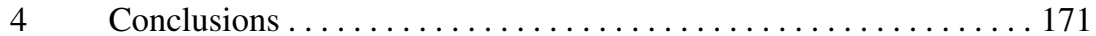

References ................................. 171 
Particle Decay in Ising Field Theory with Magnetic Field

Gesualdo Delfino

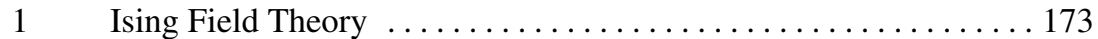

2 Evolution of the Mass Spectrum . . . . . . . . . . . . . 175

3 Particle Decay off the Critical Isotherm . . . . . . . . . . . 176

$4 \quad$ Unstable Particles in Finite Volume . . . . . . . . . . . . . . . . . . 182

References ... . . . . . . . . . . . . . . . . . . . . . . . . . . . . . . 184

Fluctuations and Large Deviations in Non-equilibrium Systems . . . . . . 187 Bernard Derrida

\begin{tabular}{|c|c|}
\hline 1 & troduction \\
\hline 2 & Large Deviation Function of the Density $\ldots \ldots \ldots \ldots \ldots$ \\
\hline 3 & Free Energy Functional \\
\hline 4 & Simple Exclusion Processes (SSEP). \\
\hline 5 & The Large Deviation Function $\mathscr{F}(\rho(x))$ for the SSEP \\
\hline 6 & The Matrix Ansatz for the Symmetric Exclusion Process \\
\hline 7 & Additivity as a Consequence of the Matrix Ansatz $\ldots \ldots \ldots \ldots$ \\
\hline 8 & Large Deviation Function of Density Profiles .......... \\
\hline 9 & $\begin{array}{l}\text { Non-locality of the Large Deviation Functional of the Density } \\
\text { and Long Range Correlations } \ldots \ldots \ldots \ldots \ldots \ldots \ldots \ldots \ldots \ldots\end{array}$ \\
\hline 10 & The Macroscopic Fluctuation Theory . \\
\hline 11 & Large Deviation of the Current $\ldots \ldots \ldots \ldots$ \\
\hline 10 & Generalized Detailed Balance and the Fluctuation Theorem ... \\
\hline 13 & Current Fluctuations in the SSEP \\
\hline 14 & The Additivity Principle......... \\
\hline
\end{tabular}

Robust Heterodimensional Cycles and Tame Dynamics

Lorenzo J. Díaz

1 Robust Heterodimensional Cycles $\ldots \ldots \ldots \ldots \ldots \ldots \ldots \ldots \ldots \ldots$

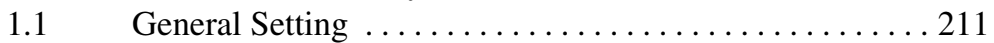

$1.2 \quad$ Basic Definitions . . . . . . . . . . . . . . . . 213

1.3 Robust Cycles at Heterodimensional Cycles . . . . . . . . 214

1.4 Questions and Consequences ............... 216

2 Cycles and Non-hyperbolic Tame Dynamics . . . . . . . . . . . 217

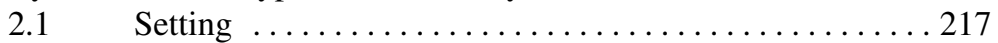

2.2 Tangencies, Heterodimensional Cycles, and Examples . . . 218

3 Robust Homoclinic Tangencies, Non-dominated Dynamics, and Heterodimensional Cycles . . . . . . . . . . . . . . . . . . . 220

$4 \quad$ Ingredients of the Proof of Theorem $2 \ldots \ldots \ldots \ldots \ldots \ldots \ldots 22$

4.1 Strong Homoclinic Intersections of Saddle-Nodes . . . . 223

$4.2 \quad$ Model Blenders ......................... 225

References .................................... 227 
Hamiltonian Perturbations of Hyperbolic PDEs: from Classification

Results to the Properties of Solutions

Boris Dubrovin

\begin{tabular}{|c|c|}
\hline 1 & Introduction \\
\hline 2 & Towards Classification of Hamiltonian PDEs \\
\hline & Deformation Theory of Integrable Hierarchies \\
\hline & $\begin{array}{l}\text { Frobenius Manifolds and Integrable Hierarchies } \\
\text { of the Topological Type } \ldots \ldots \ldots \ldots \ldots \ldots \ldots \ldots \ldots\end{array}$ \\
\hline & $\begin{array}{l}\text { Critical Behaviour in Hamiltonian PDEs, the Dispersionless } \\
\text { Case }{ }^{2} \ldots \ldots \ldots \ldots \ldots \ldots \ldots \ldots \ldots \ldots \ldots \ldots \ldots\end{array}$ \\
\hline & $\begin{array}{l}\text { Universality in Hamiltonian PDEs } \ldots \ldots \ldots \\
\text { References } \ldots \ldots \ldots \ldots \ldots \ldots \ldots \ldots \ldots\end{array}$ \\
\hline
\end{tabular}

Lattice Supersymmetry from the Ground Up

Paul Fendley and Kareljan Schoutens

References

Convergence of Symmetric Trap Models in the Hypercube

L.R.G. Fontes and P.H.S. Lima

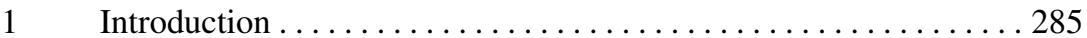

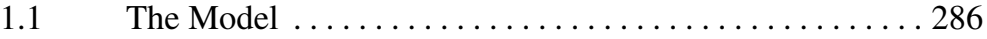

2 Convergence to the K Process . . . . . . . . . . . . . . . . . 287

$2.1 \quad$ Proof of Theorem $1 \ldots \ldots \ldots \ldots \ldots \ldots \ldots \ldots \ldots$

3 The REM-Like Trap Model and the Random Hopping Times

Dynamics for the REM ... . . . . . . . . . . . . . . . . . . . . 294

$3.1 \quad$ The REM-Like Trap Model . . . . . . . . . . . . . . . . . . . 294

3.2 Random Hopping Times Dynamics for the REM . . . . . 295

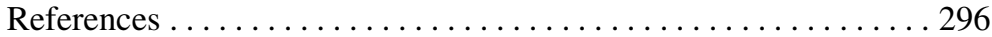

Spontaneous Replica Symmetry Breaking in the Mean Field Spin Glass

Model . . . . . . . . . . . . . . . . . . . . . . . . . . . . . . . . . . . . . 299

Francesco Guerra

$1 \quad$ Introduction . . . . . . . . . . . . . . . . . . . . . . . . . . . . . . . . . . . . . 299

2 The Mean Field Spin Glass Model. Basic Definitions . . . . . . . . 302

3 The Thermodynamic Limit . . . . . . . . . . . . . . . . . . . . . . . . 304

4 The Parisi Representation for the Free Energy . . . . . . . . . . . . 305

5 Conclusion and Outlook for Future Developments . . . . . . . . . . 309

References .................................. 310

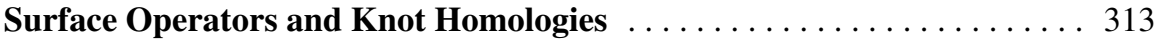

Sergei Gukov

$1 \quad$ Introduction . . . . . . . . . . . . . . . . . . . . 313

2 Gauge Theory and Categorification $\ldots \ldots \ldots \ldots \ldots \ldots \ldots \ldots \ldots \ldots$

$2.1 \quad$ Incorporating Surface Operators $\ldots \ldots \ldots \ldots \ldots \ldots \ldots$

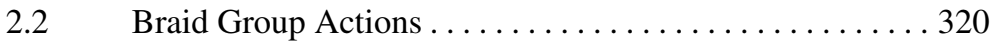


3 Surface Operators and Knot Homologies in $\mathscr{N}=2$ Gauge

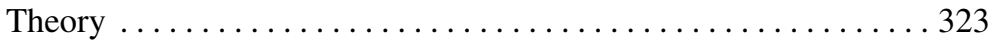

3.1 Donaldson-Witten Theory and the Equivariant Knot

Signature ... . . . . . . . . . . . . . . . . . . . . 323

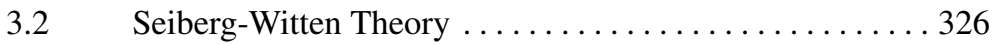

4 Surface Operators and Knot Homologies in $\mathscr{N}=4$ Gauge

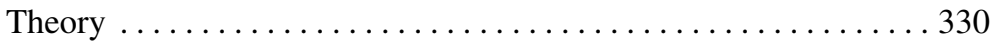

References ............................... 340

Conformal Field Theory and Operator Algebras . . . . . . . . . . . . . . . . 345

Yasuyuki Kawahigashi

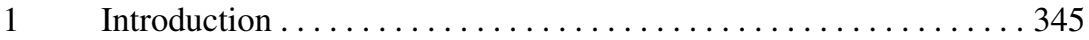

2 Conformal Quantum Field Theory ..................... 346

3 Representation Theory .......................... 349

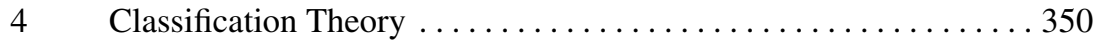

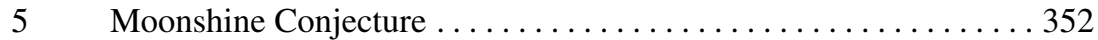

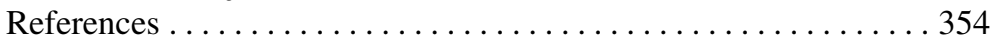

Diffusion and Mixing in Fluid Flow: A Review . . . . . . . . . . . . . . . . 357

Alexander Kiselev

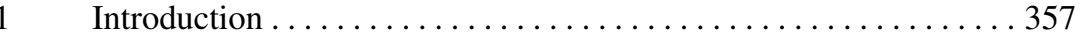

2 The Heart of the Matter . . . . . . . . . . . . . . . . . 363

3 Open Questions ............................. 367

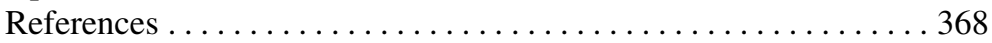

Random Schrödinger Operators: Localization and Delocalization, and

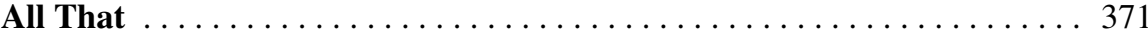

François Germinet and Abel Klein

1 Random Schrödinger Operators .................. 371

2 Basic Examples of Random Schrödinger Operators . . . . . . . . . . 372

2.1 The Anderson (Tight-Binding) Model . . . . . . . . . . . 373

2.2 The (Continuum) Anderson Hamiltonian . . . . . . . . . . . . 373

2.3 The Random Landau Hamiltonian. . . . . . . . . . . . . . . . . 373

2.4 The Poisson Hamiltonian . . . . . . . . . . . . . . . . . 374

3 The Metal-Insulator Transition . . . . . . . . . . . . . . . . . 374

4 The Spectral Metal-Insulator Transition . . . . . . . . . . . . . . . . 375

$4.1 \quad$ Anderson Localization ... . . . . . . . . . . . . . . . 375

4.2 Absolutely Continuous Spectrum .................. 377

4.3 The Spectral Metal-Insulator Transition

for the Anderson Model on the Bethe Lattice . . . . . . . . 377

5 The Dynamical Metal-Insulator Transition . . . . . . . . . . . . 378

5.1 Dynamical Localization . . . . . . . . . . . . . . . . . . 378

$5.2 \quad$ Transport Exponents . . . . . . . . . . . . . . . . . . . . 379

5.3 The Dynamical Spectral Regions ............. . 380

5.4 The Region of Complete Localization . . . . . . . . . . 381 
6 The Dynamical Transition in the Random Landau Hamiltonian . . 382 References ................................ 384

Unifying R-Symmetry in M-Theory . . . . . . . . . . . . . . . . . . . . . . . . 389 Axel Kleinschmidt

$1 \quad$ Introduction . . . . . . . . . . . . . . . . . . . . . . . 389

$2 \quad$ Kinematics..................................... 392

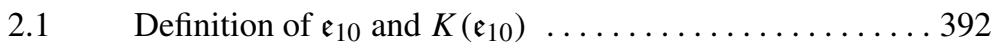

2.2 Level Decompositions for $D=11$, IIA and IIB . . . . . 393

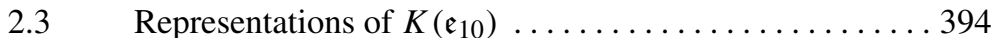

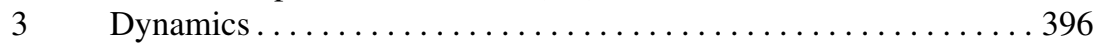

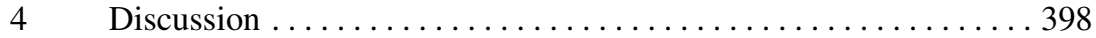

$4.1 \quad$ Remarks . . . . . . . . . . . . . . . . . . . . . . 398

$4.2 \quad$ Outlook.................................... 399

References ........................ 400

Stable Maps are Dense in Dimensional One . . . . . . . . . . . . . . 403

Oleg Kozlovski, and Sebastian van Strien

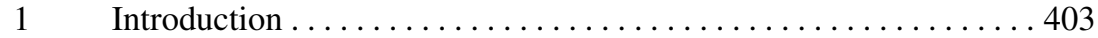

2 Density of Hyperbolicity . . . . . . . . . . . . . . . . . . 404

3 Quasi-Conformal Rigidity ...................... 405

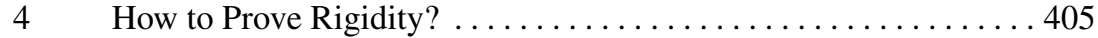

4.1 The Strategy of the Proof of QC-Rigidity . . . . . . . . 406

$5 \quad$ Enhanced Nest Construction .......................... 407

$6 \quad$ Small Distortion of Thin Annuli . . . . . . . . . . . . . . . . . . . . . . . 409

7 Approximating Non-renormalizable Complex Polynomials . . . . . . 411 References .........................4 412

Large Gap Asymptotics for Random Matrices . . . . . . . . . . . . . . . . . . 413

Igor Krasovsky

References ...........................419

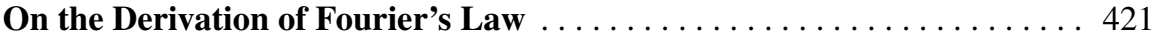

Antti Kupiainen

$1 \quad$ Introduction $\ldots \ldots \ldots \ldots \ldots \ldots \ldots \ldots \ldots \ldots \ldots \ldots \ldots \ldots \ldots \ldots \ldots$

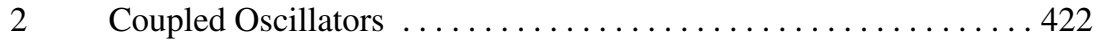

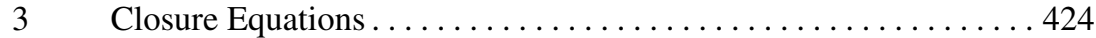

$4 \quad$ Kinetic Limit . . . . . . . . . . . . . . . . . . . . . . . . . . . . . . . . . . . 427

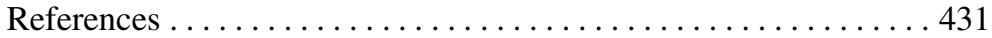

Noncommutative Manifolds and Quantum Groups . . . . . . . . . . . . . 433

Giovanni Landi

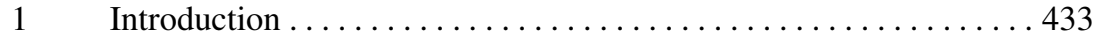

2 The Algebras and the Representations ............... 435

2.1 The Algebras of Functions and of Symmetries . . . . . . 435

2.2 The Equivariant Representation of $\mathscr{A}\left(S U_{q}(2)\right) \ldots \ldots \ldots 438$

2.3 The Spin Representation. . . . . . . . . . . . . . . . . . . 439 
3 The Equivariant Dirac Operator . . . . . . . . . . . . . . . . . . 442

4 The Real Structure . . . . . . . . . . . . . . . . . . . . . . . . . . . . 444

4.1 The Tomita Operator of the Regular Representation . . . . . 444

4.2 The Real Structure on Spinors . . . . . . . . . . . . . . . . . 445

5 The Local Index Formula for $S U_{q}(2) \ldots \ldots \ldots \ldots \ldots \ldots 47$

$5.1 \quad$ The Cosphere Bundle and the Dimension Spectrum . . . . . 448

5.2 The Local Index Formula for 3-Dimensional

Geometries ............................. . 450

$5.3 \quad$ The Pairing Between $H C^{1}$ and $K_{1} \ldots \ldots \ldots \ldots \ldots 42$

References .................................. 454

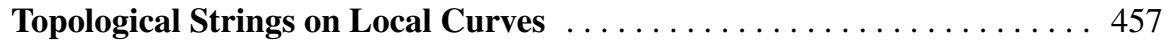

Marcos Mariño

$1 \quad$ Introduction . . . . . . . . . . . . . . . . . . . . . . . . . . . . . 457

2 Topological Strings on Local Curves ................... 459

$2.1 \quad$ A Model . . . . . . . . . . . . . . . . . . . . 459

$2.2 \quad$ Relation to Hurwitz Theory . . . . . . . . . . . . . . 460

2.3 Mirror Symmetry from Large Partitions . . . . . . . . . 462

$2.4 \quad$ Higher Genus and Matrix Models . . . . . . . . . . . . . . . . 464

3 Phase Transitions, Critical Behavior and Double-Scaling Limit . . 465

3.1 Review of Phase Transitions in Topological String

Theory .......................... 465

$3.2 \quad$ Phase Transitions for Local Curves . . . . . . . . . . . . . 467

4 Non-perturbative Effects and Large Order Behavior. . . . . . . . . . . . 469

References ......................... 472

Repeated Interaction Quantum Systems . . . . . . . . . . . . . . 475

Marco Merkli

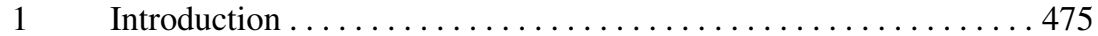

2 Deterministic Systems . . . . . . . . . . . . . . . . . . . 477

$2.1 \quad$ Mathematical Description . . .............. 477

$2.2 \quad$ Results ............................ 481

$2.3 \quad$ Asymptotic State $\ldots \ldots \ldots \ldots \ldots \ldots \ldots \ldots \ldots \ldots \ldots . \ldots \ldots 4$

2.4 Correlations \& Reconstruction of Initial State . . . . . . . 482

3 Random Systems . . . . . . . . . . . . . . . . . . . 482

3.1 Dynamics and Random Matrix Products . . . . . . . . 482

$3.2 \quad$ Results ............................ 484

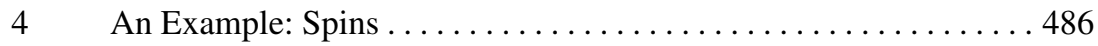

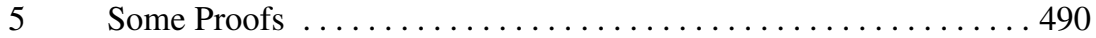

References ............................... 494

String-Localized Quantum Fields, Modular Localization, and Gauge

Theories

Jens Mund

1 The Notion of String-Localized Quantum Fields 495 
2 Modular Localization and the Construction of Free

String-Localized Fields . . . . . . . . . . . . . . . . . . . . . . . 497

3 Results on Free String-Localized Fields . . . . . . . . . . . . . . . . . . . . 499

$3.1 \quad$ Fields and Two-Point Functions . . . . . . . . . . . . . . 499

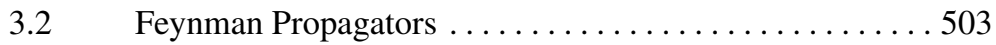

$4 \quad$ Outlook: Interacting String-Localized Fields . . . . . . . . . . . . . 504

References ............................ 507

Kinks and Particles in Non-integrable Quantum Field Theories . . . . . . . 509 Giuseppe Mussardo

Introduction . . . . . . . . . . . . . . . . . . . . . . . . . . 509

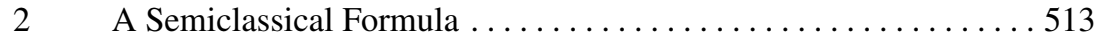

3 Symmetric Wells .............................. 515

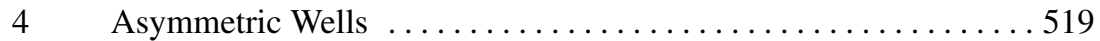

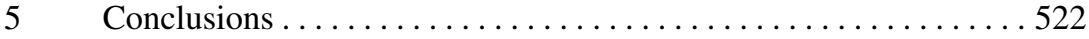

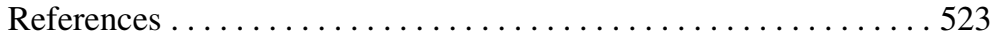

\section{Exponential Decay Laws in Perturbation Theory of Threshold}

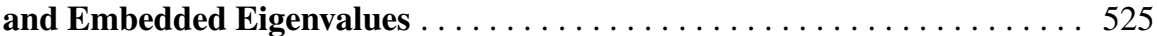

Arne Jensen and Gheorghe Nenciu

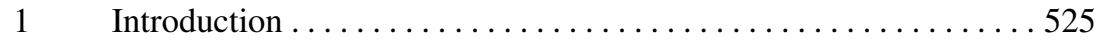

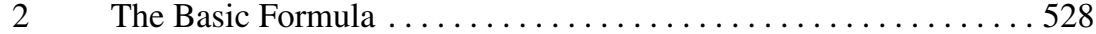

3 The Results ................................ 530

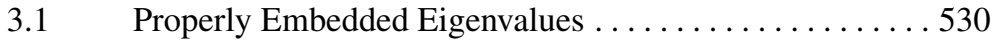

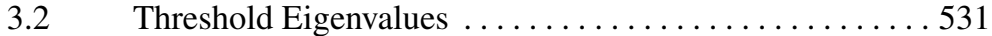

$4 \quad$ A Uniqueness Result . . . . . . . . . . . . . . . . . . . . . . . . 533

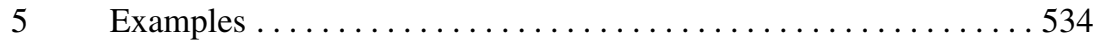

$5.1 \quad$ Example 1: One Channel Case, $v=-1 \ldots \ldots \ldots 34$

5.2 Example 2: Two Channel Case, $v=-1,1 \ldots \ldots 55$

5.3 Example 3: Two Channel Radial Case, $v \geq 3 \ldots \ldots 56$

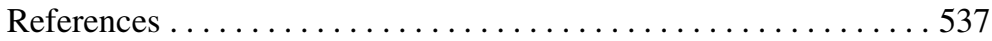

Energy Diffusion and Superdiffusion in Oscillators Lattice Networks . . . 539 Stefano Olla

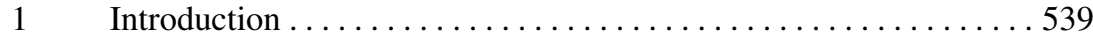

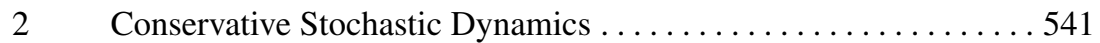

3 Diffusive Evolution: Green-Kubo Formula . . . . . . . . . . . . . . . . 544

4 Kinetic Limits: Phonon Boltzmann Equation ............... 545

5 Levy's Superdiffusion of Energy . . . . . . . . . . . . . . . . . 546

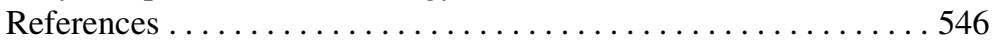

Trying to Characterize Robust and Generic Dynamics . . . . . . . . . . . . . . 549

Enrique R. Pujals

1 Introduction

2 Robust Transitivity: Hyperbolicity, Partial Hyperbolicity and Dominated Splitting 
$2.1 \quad$ Hyperbolicity ............................ 553

$2.2 \quad$ Partial Hyperbolicity . . . . . . . . . . . . . . . . . . . 554

$2.3 \quad$ Dominated Splitting ........................ 555

2.4 A General Question About "Weak Form of Hyperbolicity". ........................... 557

2.5 Robust Transitivity and Mechanisms:

Heterodimensional Cycle ..................... 557

$3 \quad$ Wild Dynamics .............................. 558

$3.1 \quad$ Wild Dynamic and Homoclinic Tangency ........... 558

3.2 Surfaces Diffeomorphisms and Beyond ............ 559

4 Generic Dynamics: Mechanisms and Phenomenas ............ 560

References ............................... 561

Dynamics of Bose-Einstein Condensates $\ldots \ldots \ldots \ldots \ldots \ldots \ldots \ldots$

Benjamin Schlein

$1 \quad$ Introduction ................................ 565

2 Heuristic Derivation of the Gross-Pitaevskii Equation . . . . . . . . 567

$3 \quad$ Main Results ............................. 571

$4 \quad$ General Strategy of the Proof and Previous Results ............ 574

$5 \quad$ Convergence to the Infinite Hierarchy ................. 576

6 Uniqueness of the Solution to the Infinite Hierarchy . . . . . . . 580

$6.1 \quad$ Higher Order Energy Estimates ................ 581

$6.2 \quad$ Expansion in Feynman Graphs .................. 583

References .....................................589

Locality Estimates for Quantum Spin Systems . . . . . . . . . . . . . . 591

Bruno Nachtergaele and Robert Sims

$1 \quad$ Introduction . ................................. 591

$2 \quad$ Lieb-Robinson Bounds.......................... 593

3 Quasi-Locality of the Dynamics ..................... 598

$4 \quad$ Exponential Clustering ...........................6 601

5 The Lieb-Schultz-Mattis Theorem ...................... 604

5.1 The Result and Some Words on the Proof ........... 605

5.2 A More Detailed Outline of the Proof ................ 607

References ...................................614

\section{On Resolvent Identities in Gaussian Ensembles at the Edge}

of the Spectrum . . . . . . . . . . . . . . . . . . . . . . . . . . . . 615

Alexander Soshnikov

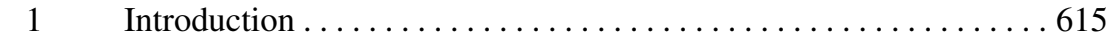

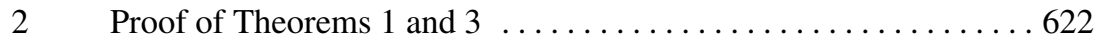

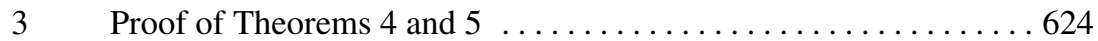

$4 \quad$ Non-Gaussian Case ................................. 625

References ...............................626 
Energy Current Correlations for Weakly Anharmonic Lattices . . . . . . . 629

Herbert Spohn

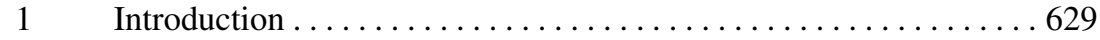

2 Anharmonic Lattice Dynamics . . . . . . . . . . . . . . . . . . . 630

3 Energy Current Correlations . . . . . . . . . . . . . . . . 633

4 The Linearized Collision Operator . . . . . . . . . . . . . . 637

5 Gaussian Fluctuation Theory ........................ 639

References ....................................6640

Metastates, Translation Ergodicity, and Simplicity of Thermodynamic

States in Disordered Systems: an Illustration . . . . . . . . . . . . . . . . 643

Charles M. Newman and Daniel L. Stein

$1 \quad$ Introduction . . . . . . . . . . . . . . . . . . . . . 643

2 The Sherrington-Kirkpatrick Model and the Parisi Replica

Symmetry Breaking Solution . . . . . . . . . . . . . . . . . . 644

3 Open Problems . . . . . . . . . . . . . . . . . . . . . . . 645

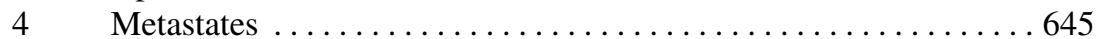

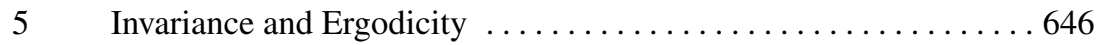

6 A Strategy for Rigorous Studies of Spin Glasses . . . . . . . . . . . . 647

$7 \quad$ Summary . . . . . . . . . . . . . . . . . . . . . . 651

References ...........................651

Random Matrices, Non-intersecting Random Walks, and Some Aspects

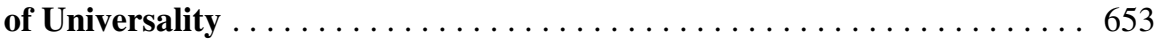

Toufic M. Suidan

$1 \quad$ Introduction . . . . . . . . . . . . . . . . . . . . . . . . 653

1.1 Selected Basic Facts from Random Matrix Theory . . . . . 654

1.2 The Karlin-McGregor Formula . . . . . . . . . . . . . . 654

2 The Models ............................... 655

2.1 Longest Increasing Subsequence of a Random

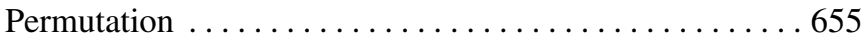

$2.2 \quad$ ABC-Hexagon ..........................665 65

$2.3 \quad$ Last Passage Percolation . . . . . . . . . . . . . 657

$2.4 \quad$ Non-intersecting Brownian Motion . . . . . . . . . . . 660

$3 \quad$ Universality . . . . . . . . . . . . . . . . . . . . 661

$3.1 \quad$ Last Passage Percolation . . . . . . . . . . . . . . . . 662

$3.2 \quad$ Non-intersecting Random Walks . . . . . . . . . . . . 662

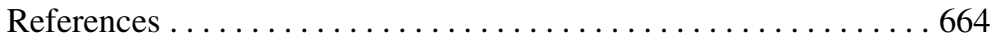

Homogenization of Periodic Differential Operators as a Spectral

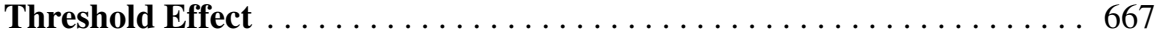

Mikhail S. Birman and Tatiana A. Suslina

$1 \quad$ Introduction . . . . . . . . . . . . . . . . . . . . . . . . 667

2 Periodic DO's. The Effective Matrix ................. 668

3 Homogenization of Periodic DO's. Principal Term of Approximation for the Resolvent . . . . . . . . . . . . . . 670 
4 More Accurate Approximation for the Resolvent

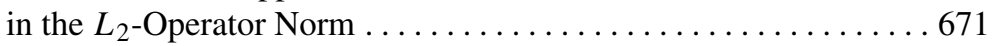

$5 \quad\left(L_{2} \rightarrow H^{1}\right)$-Approximation of the Resolvent. Approximation

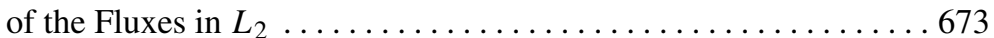

6 The Method of Investigation . . . . . . . . . . . . . . . . . . . 674

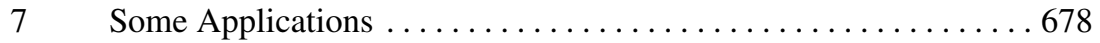

8 On Further Development of the Method ...............661

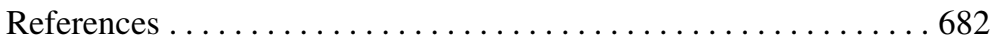

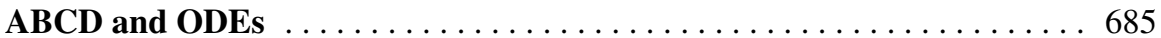

Patrick Dorey, Clare Dunning, Davide Masoero, Junji Suzuki and Roberto Tateo

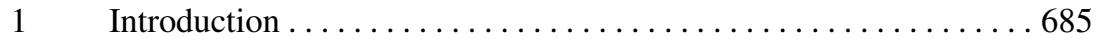

2 Bethe Ansatz for Classical Lie Algebras . . . . . . . . . . . . 688

3 The Pseudo-Differential Equations . . . . . . . . . . . . . . . 689

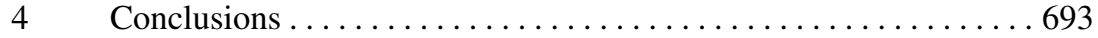

References ............................694

Nonrational Conformal Field Theory . . . . . . . . . . . . . . . . . . . . . . . 697

Jörg Teschner

$1 \quad$ Introduction . . . . . . . . . . . . . . . . . . . . 697

2 Constraints from Conformal Symmetry .................6699

2.1 Motivation: Chiral Factorization of Physical Correlation

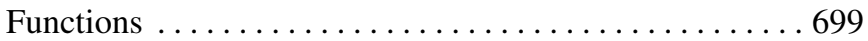

$2.2 \quad$ Vertex Algebras ...................... 700

2.3 Representations of Vertex Algebras ............. 701

2.4 Conformal Blocks ..................... 701

2.5 Correlation Functions vs. Hermitian Forms . . . . . . . 703

3 Behavior Near the Boundary of Moduli Space . . . . . . . . . . . . . 704

$3.1 \quad$ Gluing of Riemann Surfaces . . . . . . . . . . . . . 705

$3.2 \quad$ Gluing of Conformal Blocks $\ldots \ldots \ldots \ldots \ldots \ldots \ldots \ldots$

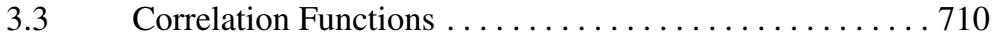

3.4 Conformal Blocks as Matrix Elements . . . . . . . . . 712

4 From one Boundary to Another................. 713

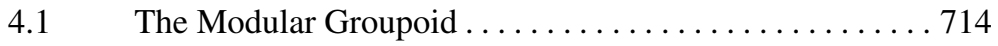

4.2 Representation of the Generators on Spaces

of Conformal Blocks .................. 717

4.3 Representation of the Relations on Spaces of Conformal

Blocks . . . . . . . . . . . . . . . . . . . . . . . 718

$5 \quad$ Notion of a Stable Modular Functor . . . . . . . . . . . . . . . 719

5.1 Towers of Representations of the Modular Groupoid . . . 719

$5.2 \quad$ Unitary Modular Functors $\ldots \ldots \ldots \ldots \ldots \ldots \ldots \ldots \ldots$

5.3 Similarity of Modular Functors . . . . . . . . . . 722

$5.4 \quad$ Friedan-Shenker Modular Geometry . . . . . . . . . 722

6 Example of a Nonrational Modular Functor . . . . . . . . . . . 723 
6.1 Unitary Positive Energy Representations of the Virasoro Algebra ...................... 724

6.2 Construction of Virasoro Conformal Blocks in Genus Zero............................... 725

$6.3 \quad$ Factorization Property .................. 726

6.4 The Hilbert Space Structure . . . . . . . . . . . . . . 727

$6.5 \quad$ Extension to Higher Genus . . . . . . . . . . . . . . . . 728

$6.6 \quad$ Remarks . . . . . . . . . . . . . . . . . . . . 729

7 Existence of a Canonical Scalar Product? . . . . . . . . . . . . . . . . 729

7.1 Existence of a Canonical Hermitian Form

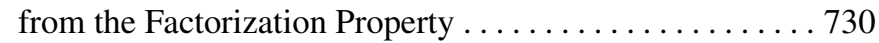

$7.2 \quad$ Unitary Fusion . . . . . . . . . . . . . . . . . 732

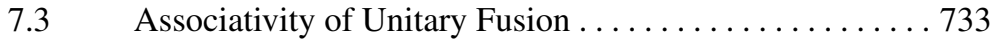

$7.4 \quad$ Discussion ......................... 735

$8 \quad$ Outlook ................................... 735

8.1 Modular Functors from W-algebras and Langlands Duality ............................ 735

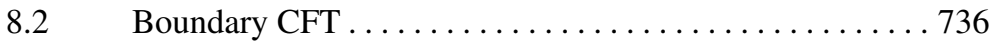

$8.3 \quad$ Nonrational Verlinde Formula? . . . . . . . . . . 736

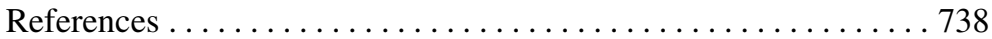

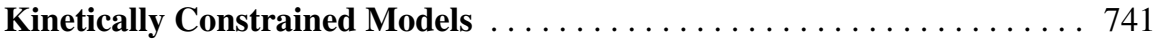

Nicoletta Cancrini, Fabio Martinelli, Cyril Roberto and Cristina Toninelli

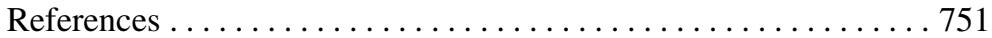

The Distributions of Random Matrix Theory and their Applications . . . 753 Craig A. Tracy and Harold Widom

1 Random Matrix Models: Gaussian Ensembles . . . . . . . . . . . 753

1.1 Largest Eigenvalue Distributions $F_{\beta}$. Painlevé II Representations ....................... 754

$1.2 \quad$ Next-Largest, Next-Next Largest, Etc. Eigenvalue

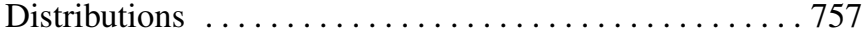

$2 \quad$ Universality Theorems . . . . . . . . . . . . . . . . . . . . . . . 757

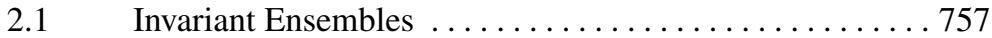

$2.2 \quad$ Wigner Ensembles.......................... . . 759

3 Multivariate Statistical Analysis . . . . . . . . . . . . . . . . . . . . . 759

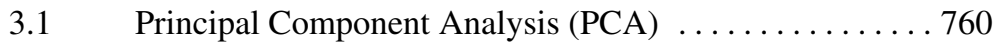

3.2 Testing the Null Hypothesis . . . . . . . . . . . . . . . . 760

3.3 Spiked Populations: BBP Phase Transition .......... 761

$4 \quad$ Conclusions .............................. 763

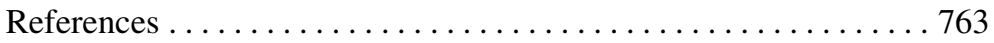

Hybrid Formalism and Topological Amplitudes . . . . . . . . . . . 767

Jürg Käppeli and Stefan Theisen and Pierre Vanhove

$1 \quad$ Introduction . . . . . . . . . . . . . . . . . . . . . . . 767

2 Compactified String Theory in RNS and Hybrid Variables . . . . . 768 
$2.1 \quad$ Hybrid Variables . . . . . . . . . . . . . . . . 768

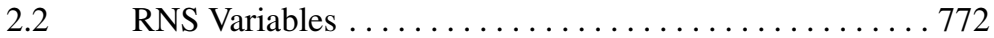

2.3 Field Redefinition from RNS to Hybrid Variables . . . . . . 773

$2.4 \quad$ Physical State Conditions and $\mathscr{N}=4$-embeddings $\ldots . .776$

$2.5 \quad$ Massless Vertex Operators ................... . 778

3 Amplitudes and Correlation Functions .................. 780

$3.1 \quad$ Amplitudes . . . . . . . . . . . . . . . . . . 780

3.2 Correlation Functions of Chiral Bosons . . . . . . . . . . . 782

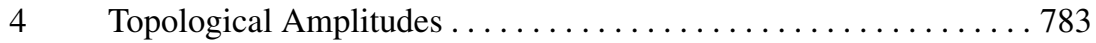

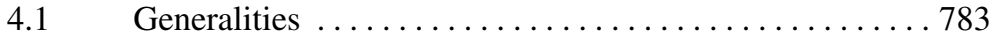

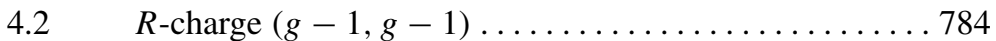

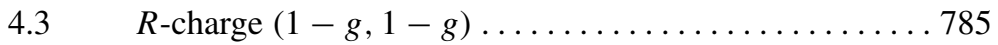

$4.4 \quad R$-charges $(g-1,1-g)$ and $(1-g, g-1) \ldots \ldots \ldots 787$

$4.5 \quad$ Summary of the Amplitude Computation . . . . . . . . . . 788

A Appendix: Conventions and Notations ................ 789

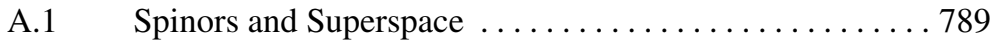

A.2 Hybrid Variables and $\mathscr{N}=2$ Algebra ............ 790

A.3 The Integrated Vertex Operator ... . . . . . . . . . . . . 791

B Appendix: Mapping the RNS to the Hybrid Variables . . . . . . . . . 792

B.1 Field Redefinition from RNS to Chiral GS Variables . . . 792

B.2 Similarity Transformation Relating Chiral GS

to Hybrid Variables . . . . . . . . . . . . . . . . . . 792

B.3 Hermitian Conjugation of the Hybrid Variables . . . . . . . 793

B.4 Hermitian Conjugation of the RNS Variables . . . . . . . . 794

C Appendix: Vertex Operators . . . . . . . . . . . . . . . 797

C.1 Massless RNS Vertex Operators ................ 797

C.2 Universal Massless Multiplets ................. 799

C.3 Compactification Dependent Massless Multiplets . . . . . . 799

References ............................ 802

Quantum Phases of Cold Bosons in an Optical Lattice . . . . . . . . . . . . 805

Michael Aizenman, Elliot H. Lieb, Robert Seiringer, Jan Philip Solovej and Jakob Yngvason

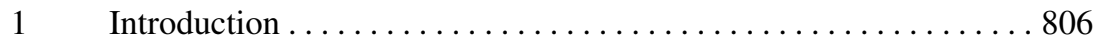

2 Reflection Positivity ....................... 810

3 Proof of BEC for Small $\lambda$ and $T \ldots \ldots \ldots \ldots \ldots \ldots \ldots \ldots \ldots \ldots \ldots$

4 Absence of BEC and Mott Insulator Phase ..... . . . . . . . . 816

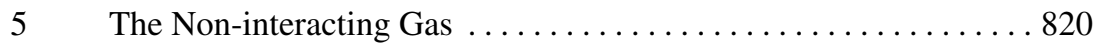

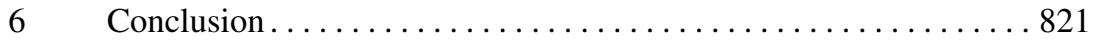

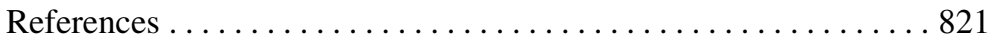

Random Walks in Random Environments in the Perturbative Regime . . 823 Ofer Zeitouni

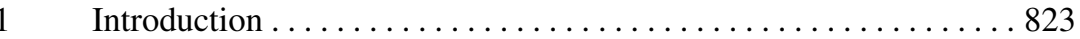

2 Local Limits for Exit Measures . . . . . . . . . . . . . . 825

References ........................... 826 
Appendix: Complete List of Abstracts

YRS and XV ICMP

1 Young Researchers Symposium Plenary Lectures . . . . . . . . . 827

2 XV International Congress on Mathematical Physics Plenary Lectures . . . . . . . . . . . . . . . . . . . . . . . . 830

3 XV International Congress on Mathematical Physics Specialized Sessions ................................ 835

$3.1 \quad$ Condensed Matter Physics $\ldots \ldots \ldots \ldots \ldots \ldots \ldots \ldots . \ldots \ldots$

3.2 Dynamical Systems . . . . . . . . . . . . . . 836

3.3 Equilibrium Statistical Mechanics . . . . . . . . . . . 839

3.4 Non-equilibrium Statistical Mechanics . . . . . . . . . . . . 840

$3.5 \quad$ Exactly Solvable Systems . . . . . . . . . . . . . . . 843

$3.6 \quad$ General Relativity . . . . . . . . . . . . . . . . . . . 844

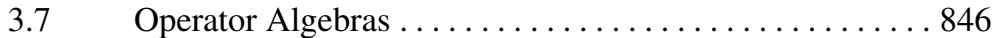

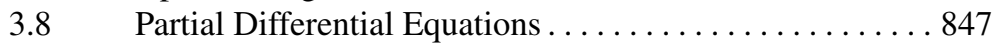

3.9 Probability Theory $\ldots \ldots \ldots \ldots \ldots \ldots \ldots \ldots \ldots \ldots \ldots$

3.10 Quantum Mechanics ........................ . 850

3.11 Quantum Field Theory ................. 852

3.12 2D Quantum Field Theory ................. 854

3.13 Quantum Information ...................... 855

3.14 Random Matrices . . . . . . . . . . . . . . . . . . . . 858

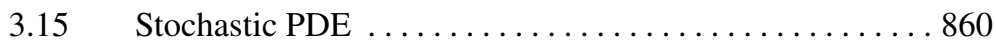

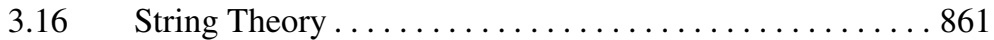

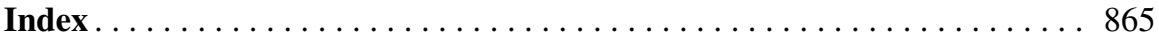

